\title{
INFLUÊNCIA DA SOMA TÉRMICA E DA CHUVA DURANTE O DESENVOLVIMENTO DE LARANJAS-'VALÊNCIA' E 'NATAL' NA RELAÇÃO ENTRE SÓLIDOS SOLÚVEIS E ACIDEZ E NO ÍNDICE TECNOLÓGICO DO SUCO ${ }^{1}$
}

\author{
CLOVIS ALBERTO VOLPE², EDGAR RICARDO SCHÖFFEL ${ }^{3}$, JOSÉ CARLOS BARBOSA²
}

\begin{abstract}
RESUMO - Este trabalho foi realizado com o objetivo de conhecer a influência que algumas variáveis meteorológicas exercem na razão entre sólidos solúveis totais e acidez total titulável ("ratio") e no índice tecnológico dos frutos da primeira florada das laranjeiras'Natal' e 'Valência', na região de Bebedouro-SP, mediante a utilização de métodos estatísticos de regressão. Foram utilizados dados de amostragens de rotina para o processamento industrial durante 4 anos, os quais permitiram desenvolver equações de regressão linear e quadrática, com a soma térmica (graus-dia) como variável independente, e de regressão múltipla, utilizando graus-dia e chuva como variáveis independentes. A equação de melhor ajuste para o índice tecnológico foi a quadrática, enquanto para o "ratio" a equação linear apresentou o melhor ajuste. A temperatura do ar, representada por graus-dia, foi a variável que exerceu maior influência nos indicadores de qualidade dos frutos.
\end{abstract}

Termos de indexação: graus-dia, temperatura, chuva, qualidade do suco.

\section{INFLUENCE OF THE ACCUMULATED HEAT UNIT AND RAINFALL ON THE RATIO AND TECHNOLOGICAL INDEX OF SWEET ORANGES 'VALÊNCIA' AND 'NATAL'}

\begin{abstract}
This study aimed to know the influence of some meteorological variables on the ratio and technological index for oranges provided from 'Natal' and 'Valencia' orchards, from field plots, located at Bebedouro - São Paulo - Brazil. The quality indicators, ratio and technological index, were obtained from routine processing plant tests. These parameters were related to the meteorological variables: degree-day and rainfall. In order to determine which independent variable had a stronger influence on the fruit quality indicators, single and multiple linear regressions analysis were applied. The results have shown that technological index is better described by a quadratic function, and ratio is better described by a linear regression, as function of independent variables. The statistical analysis have indicated that the air temperature, expressed by accumulated degree day, is the meteorological aspect that had greater influence in fruit quality indicators.
\end{abstract}

Index terms: degree-day, temperature, rainfall, juice quality.

\section{INTRODUÇÃO}

A produção de suco de laranja com alta qualidade necessita de frutos com alta qualidade, a qual é avaliada através das suas características químicas e físicas. As características físicas e químicas dos frutos variam durante o período de maturação, e essa variação depende, entre outros fatores, das condições meteorológicas durante a formação e maturação dos frutos.

O rendimento industrial é dado pelo índice tecnológico que considera as características físicas e químicas do fruto, enquanto o método utilizado para determinar a maturidade e a época da colheita dos frutos de laranja, é a razão entre as porcentagens de sólidos solúveis totais (SST) e de acidez total titulável (ATT), conhecida como índice de maturidade (SST/ATT) ou, simplesmente, "ratio". O índice tecnológico, além de indicador da maturidade, pode ser utilizado como indicador da qualidade do fruto (Sinclair, 1984; Soule e Grierson, 1986).
Na Califórnia, utiliza-se "ratio" de, no mínimo, 8 para o consumo do fruto "in natura", e "ratio" igual a 10 para frutos destinados à fabricação de suco concentrado congelado (SLCC). Na Flórida, geralmente, os consumidores preferem o suco de laranja com "ratio" entre 15 e 18, e a indústria começa a processar os frutos quando estes atingem "ratio" igual a 13 (Kimball, 1991). No Brasil, apesar do consumo de SLCC ser pequeno ( $5 \%$ do total produzido), verifica-se a preferência por sucos com "ratio" acima de 14. Todavia, o processamento pode começar quando o "ratio" alcança 12 - 13, embora o preferido pelas indústrias esteja entre 15 e 18 (Marchi, 1993).

Albrigo (1990) demonstrou que 60\% a 70\% da variabilidade entre anos agrícolas para o índice tecnológico (kg de sólidos solúveis por caixa) e o total de sólidos solúveis no suco podem ser devido às variações nas temperaturas do ar e às chuvas durante a indução floral e o período de diferenciação, antes da florada.

Chen (1990), na Califórnia e Flórida, mostrou que o

1 (Trabalho 158/2001). Recebido: 03/10/2001. Aceito para publicação: 25/06/2002.

2 Eng $^{\text {}}$ Agr $^{\circ}$, Livre Docente, Prof. Dep. Ciências Exatas da FCAV/UNESP. 14884-900, Jaboticabal-SP. Tel (16)32092625

3 Eng $^{\circ}$ Agr $^{\circ}$, Dr., Pesquisador do Departamento de Ciências Exatas da FCAV/UNESP, bolsista de Pós-Doutorado da FAPESP. Jaboticabal-SP, 14884900

Rev. Bras. Frutic., Jaboticabal - SP, v. 24, n. 2, p. 436-441, agosto 2002 
acúmulo de graus-dia pode representar o acúmulo de sólidos solúveis e o aumento no índice de maturidade do suco a partir do mês de julho. Na região de Bebedouro-SP, em 5 anos de estudo, Volpe (1992) verificou para a laranja-'Pêra', com idade superior a 10 anos, altos coeficientes de correlação entre o "ratio" e o acúmulo de graus-dia, com temperatura base de $13^{\circ} \mathrm{C}$, sendo grande a variabilidade da correlação de ano para ano.

As análises de regressão linear simples, quadráticas e múltiplas, entre as características químicas e físicas dos frutos e as variáveis climáticas, têm sido usadas na obtenção de modelos de previsão de colheita, no estabelecimento de curvas de maturação e nos estudos que procuram estabelecer a importância dos elementos meteorológicos na maturação do fruto (Kimball, 1984; Nuñes e Iglesias, 1991). Para Volpe et al. (2000), a equação de regressão quadrática com a variável independente graus-dia acumulados é a que apresenta o melhor ajuste com os indicadores de qualidade, acidez e sólidos solúveis, dos frutos de laranjeiras-'Natal' e 'Valência'.

O objetivo do presente trabalho foi determinar o grau de influência das variáveis meteorológicas, soma térmica (grausdia) e chuva, no índice de maturidade ("ratio") e no índice tecnológico dos frutos da primeira florada de laranjeiras-'Natal' e 'Valência' na região de Bebedouro-SP, utilizando-se de dados de amostragens de rotina para processamento industrial.

\section{MATERIAL E MÉTODOS}

Foram utilizados pomares de laranjeiras-'Valência' e 'Natal' (Citrus sinensis, L. Osbeck), enxertadas em limoeiro- 'Cravo' (Citrus limonia, Osbeck), localizados na região de Bebedouro-SP. As plantas foram cadastradas conforme o ano de plantio, e divididas em 3 categorias de idade: (1) plantas com idade de 3 a 5 anos; (2) plantas com idade entre 6 e 10 anos; e (3) plantas com idade superior a 10 anos.

Foram estudadas as curvas de maturação dos frutos da primeira florada de laranjeiras das variedades tardias Valência e Natal, durante 4 anos, 1994, 1996, 1997 e 1998, que correspondem, respectivamente, às safras 94-95; 96-97; 97-98 e 98-99. Os frutos dessas variedades, na indústria de suco, são analisados conjuntamente, pois não ocorrem grandes diferenças nas curvas de maturação dessas duas variedades.

As amostragens de frutos iniciaram-se, em todos os anos, no mês de abril, e estenderam-se até o mês da colheita, novembro ou dezembro. Em cada pomar, foram demarcadas oito árvores para o acompanhamento da maturação dos frutos, e, em cada árvore, foram coletados, mensalmente, cinco frutos para o acompanhamento da curva de maturação.

Os teores de sólidos solúveis totais $(\mathrm{g} / 100 \mathrm{ml})$ foram determinados por refratometria a $20^{\circ} \mathrm{C}$, e a acidez total titulável (\% de ácido cítrico) foi determinada por titulometria, usando-se como titulante $\mathrm{NaOH}$ a $0,3125 \mathrm{M}$ (Read et al., 1986).

O "ratio" foi calculado pela relação entre os teores de sólidos solúveis totais e a acidez total titulável, enquanto o índice tecnológico (sólidos solúveis/caixa) foi obtido pela expressão:

$$
\mathrm{IT}=(\mathrm{RS} . \mathrm{SST} \cdot 40,8) \cdot 10^{-4}
$$

onde, IT é o índice tecnológico; RS, em \%, é o rendimento em suco; SST, em g/100 ml, é o teor de sólidos solúveis; e 40,8 é peso padrão da caixa de colheita de laranja $(\mathrm{kg})$.
Os dados diários de temperaturas máxima e mínima, e de chuva dos anos de 1993 a 1998, foram obtidos na Estação Meteorológica da Estação Experimental de Citricultura de Bebedouro, cujas coordenadas geográficas são $20^{\circ} 58^{\prime} 18^{\prime}$ 'S, 48²8’11”W, 600 m.

A soma de graus-dia para os períodos compreendidos entre o florescimento e a data de amostragem foi obtida de acordo com a expressão:

$$
\mathrm{GM}=\sum_{d}^{n}(T m-T b)
$$

onde, , d é o dia considerado do florescimento e n é a data da amostragem; $\mathrm{Tm}, \mathrm{em}^{\circ} \mathrm{C}$, é a temperatura média diária; $\mathrm{Tb}, \mathrm{em}^{\circ} \mathrm{C}$, é a temperatura base inferior, igual a $13,0^{\circ} \mathrm{C}$ (Monselise, 1986). A Tm foi determinada através da média aritmética entre as temperaturas máxima e mínima diárias.

Os dados obtidos foram ajustados a vários modelos de regressão, e o grau de ajuste foi medido através do teste $\mathrm{F}$ da análise de variância, e do coeficiente de determinação ajustado ao número de parâmetros $\left(\mathrm{R}^{2} \mathrm{AJ}\right)$. Para a análise dos dados, utilizou-se o "software" Statistical Analysis System (SAS), através do Procedimento Regressão (PROC REG). Destaca-se que as análises de regressão foram feitas considerando-se todas as amostras, e não a média dessas. O número de amostras variou de 117 (1998, idade 1) a 828 (1994, idade 3).

Em todas as equações, a variável dependente representa as características "ratio" ou índice tecnológico, e a variável independente representa, na equação linear e quadrática, graus-dia acumulados, do florescimento até a data da amostragem. Na regressão múltipla, as variáveis independentes representam a soma de graus-dia e o acúmulo de chuva, do florescimento até a data da amostragem.

\section{RESULTADOS E DISCUSSÃO}

As temperaturas médias anuais dos anos em estudo, de 1993 a 1998, variaram de $23,5^{\circ} \mathrm{C}$, em 1993 , a 24,0 ${ }^{\circ} \mathrm{C}$, em 1994, e a temperatura média mensal variou de $19,4^{\circ} \mathrm{C}$, em junho de 1996 , a $27,7^{\circ} \mathrm{C}$, em janeiro de 1998 . Nos meses mais frios, junho e julho, a temperatura média ficou entre $19,4^{\circ} \mathrm{C}$, em 1996 , e $20,6^{\circ} \mathrm{C}$, em 1997. As maiores temperaturas máximas médias mensais variaram de $27,1^{\circ} \mathrm{C}$ a $35,3^{\circ} \mathrm{C}$. Nos meses de maio, junho e julho, durante as fases de pré-florescimento, de crescimento e de maturação do fruto, as temperaturas mínimas médias mensais ficaram abaixo de $13,0^{\circ} \mathrm{C}$, alcançando valores superiores a $18,0^{\circ} \mathrm{C}$ de outubro a março, período de crescimento e maturação dos frutos, não sendo verificado dano de geada em pomares de laranja nos anos do estudo.

As precipitações dos meses de junho, julho e agosto foram inferiores a $30 \mathrm{~mm}$, exceto nos ano de 1994 e 1997, quando, em junho, o total de chuva foi de 44 e $157 \mathrm{~mm}$, respectivamente, e, em agosto de 1998, com um total de $88,6 \mathrm{~mm}$. O ano menos chuvoso foi o ano de 1994, com 1146 m, e o mais chuvoso foi o de 1998, com $1701 \mathrm{~mm}$.

As regressões entre "ratio" e as variáveis meteorológicas graus-dia e chuva foram todas estatisticamente significativas pelo teste $\mathrm{F}(\mathrm{p}<0,01)$, independentemente do ano e categoria de idade, indicando que existe uma relação funcional 
entre o "ratio" e as variáveis estudadas. Não são apresentados os valores obtidos para a regressão quadrática, com graus-dia como variável independente, porque o ajuste dessas equações foi demasiadamente pequeno, em relação ao ajuste das outras equações.

Os valores do coeficiente de determinação ajustado das regressões encontram-se na Tabela 1 . De acordo os valores de $\mathrm{R}^{2} \mathrm{AJ}$ da regressão linear simples, no pior ajuste, a variável grausdia explicou cerca de $69 \%$ da variação do "ratio", no melhor ajuste, $88 \%$, e que, em média explicou $82 \%$. Forte correlação linear entre "ratio" e graus-dia acumulados foi observada por Kimball (1984) em laranja-'Valência', na Califórnia, com $\mathrm{R}^{2}$ atingindo até 0,96 .

A análise de regressão múltipla com graus-dia e chuva, praticamente, não alterou os valores de $\mathrm{R}^{2} \mathrm{AJ}$. Isto mostra que a inclusão da chuva não provocou grandes benefícios ao ajuste da equação de regressão.

Correlação significativa entre o "ratio" e o mês de análise, obedecendo a uma função linear positiva, foi verificada por vários autores, entre os quais Chitarra e Chitarra (1979) e Chitarra e Campos (1981).

Através das Figuras 1; 2 e 3, verificam-se diferenças entre os anos estudados nas equações de regressão. Isto, provavelmente, se deve às diferenças meteorológicas ocorridas entre os anos.

Essa variabilidade de ano para ano tem sido relatada na literatura (Kimball, 1984; Volpe, 1992). As variações meteorológicas de ano para ano, que alteram o comportamento da variação dos sólidos solúveis e, principalmente, dos ácidos, causam mudanças no valor do "ratio" (Nuñez et al., 1987; Volpe et al., 2000). Um fator importante que influi no "ratio", é o vigor da planta, representado pela carga de frutos durante a maturação, que pode, inclusive, ser avaliada através da produtividade (caixas/árvore). Eles podem ser utilizados para o ajuste das inclinações das retas, de diferentes anos, pois elas refletem a taxa de desenvolvimento do "ratio".

Os resultados obtidos no presente trabalho estão em concordância com Di Giorgi et al. (1991). Eles relatam que a evolução do "ratio" de laranjas-doces no Estado de São Paulo, em parte, pode ser explicado pela relação porta-enxerto/copa, idade das árvores, florada e produtividade, mas que, principalmente, o clima é de extrema importância na variação de ano para ano.

Não se verificou diferença significativa entre as inclinações das retas das categorias de idades 2 ( 6 a 10 anos) e 3 (> 10 anos). Elas foram maiores nessas idades, e diferiram da categoria 1 (plantas mais jovens). Mesmo assim, as plantas jovens atin- gem o "ratio" 12 antes das plantas mais velhas, em virtude de que elas apresentam um maior crescimento inicial dos frutos, como pode ser verificado pelos interceptos. Isto está de acordo com Di Giorgi et al. (1991), que verificaram maturação mais precoce dos frutos de plantas jovens. Baseado neste fato, a colheita das plantas jovens é planejado para o início da safra, cerca de 20 dias antes da colheita das idades 2 e 3 .

As regressões entre o índice tecnológico e as variáveis meteorológicas graus-dia e chuva foram todas estatisticamente significativas pelo teste $\mathrm{F}(\mathrm{p}<0,01)$, indicando que existe uma relação funcional entre o índice tecnológico e as variáveis meteorológicas estudadas.

Observa-se, através dos dados de $\mathrm{R}^{2} \mathrm{AJ}$ da Tabela 2, que, para a regressão linear simples, a variável graus-dia explicou em média $52 \%$ da variação do índice tecnológico. Os valores de $\mathrm{R}^{2} \mathrm{AJ}$ da regressão quadrática, com graus-dia como variável independente, variaram entre 0,811 a 0,372 , com valor médio de 0,600 , apresentando, assim, melhor ajuste.

A inclusão da chuva como variável independente, juntamente com graus-dia, na regressão múltipla, não melhorou o ajuste da regressão. Neste caso, o $\mathrm{R}^{2} \mathrm{AJ}$ variou de 0,710 a 0,443 , com valor médio de 0,566 .

São raros, na literatura, trabalhos que correlacionam o índice tecnológico com as variáveis climáticas. Na Califórnia, Albrigo (1990), analisando essa característica ao longo de 20 anos, verificou que o índice tecnológico apresentou uma variabilidade de ano para ano de $1,132 \mathrm{~kg}$, sendo essas oscilações explicadas em $70 \%$ pelos efeitos da chuva e da temperatura.

$\mathrm{O}$ índice tecnológico não constitui o resultado de uma medida e, sim, de um cálculo, cujos parâmetros são o teor de sólidos solúveis e o rendimento em suco; portanto, ele é influenciado por todos os fatores que afetam o comportamento desses parâmetros.

Nas Figuras 4; 5 e 6, respectivamente, para as idades 1;2 e 3, estão representadas as equações e os gráficos das regressões linear e quadrática, onde se percebe que houve diferenças nos parâmetros de ajustes entre anos e categorias de idade.

Do ponto de vista prático, o índice tecnológico constitui um parâmetro seletivo para a escolha de pomares a serem colhidos, pois o planejamento da colheita visa à otimização dos sólidos solúveis e palatabilidade do fruto (Marchi, 1993). Dessa forma, equações de regressão de melhor ajuste devem ser obtidas, quando se deseja fazer previsão do índice tecnológico. De acordo com os resultados obtidos nesse trabalho, a temperatura do ar, representada por graus-dia, deve ser considerada nos modelos de previsão do índice tecnológico.
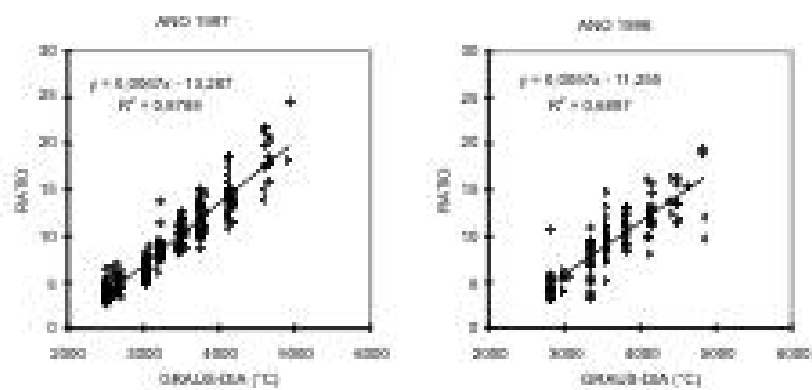

FIGURA 1 - Curvas da equação de regressão linear para o "ratio", em função de graus-dia acumulados por ano, em frutos da primeira florada de laranjeiras-'Valência' e 'Natal', com 3 a 5 anos de idade. Bebedouro-SP.

Rev. Bras. Frutic., Jaboticabal - SP, v. 24, n. 2, p. 436-441, agosto 2002 
TABELA 1 - Valores dos coeficientes de determinação ajustados $\left(\mathrm{R}^{2} \mathrm{AJ}\right)$ das regressões linear simples e múltiplas para o "ratio", por ano e categoria de idade, para laranjas-'Valência' e 'Natal', em Bebedouro-SP.

\begin{tabular}{cccc}
\hline ANO & $\begin{array}{c}\text { IDADE } \\
\text { (anos) }\end{array}$ & GD & GD, C \\
\cline { 2 - 4 } & $3-5$ & Linear & Múltipla \\
\hline \multirow{2}{*}{1994} & $6-10$ & 0,838 & 0,839 \\
& $>10$ & 0,866 & 0,874 \\
& $3-5$ & 0,846 & 0,846 \\
1996 & $6-10$ & 0,831 & 0,831 \\
& $>10$ & 0,814 & 0,813 \\
& $3-5$ & 0,813 & 0,812 \\
& $6-10$ & 0,878 & 0,879 \\
& $>10$ & 0,832 & 0,832 \\
& $3-5$ & 0,882 & 0,885 \\
& $6-10$ & 0,686 & 0,691 \\
\hline MÉDIA & $>10$ & 0,731 & 0,732 \\
\hline
\end{tabular}

$\overline{\mathrm{GD}}=$ graus-dia; $\mathrm{C}$ = chuva.

TABELA 2 - Valores dos coeficientes de determinação ajustados $\left(\mathrm{R}^{2} \mathrm{AJ}\right)$ das regressões linear simples, quadrática e múltiplas para o índice tecnológico, por ano, categoria de idade e florada, para laranjas-'Valência' e 'Natal', em Bebedouro-SP.

\begin{tabular}{ccccc}
\hline ANO & IDADE & GD & GD & GD, C \\
\cline { 2 - 5 }$($ anos $)$ & $3-5$ & Linear & Quadrática & Múltipla \\
\hline \multirow{2}{*}{1994} & $6-10$ & 0,621 & 0,662 & 0,635 \\
& $>10$ & 0,683 & 0,770 & 0,701 \\
1996 & $3-5$ & 0,695 & 0,811 & 0,710 \\
& $6-10$ & 0,304 & 0,372 & 0,443 \\
1997 & $>10$ & 0,340 & 0,447 & 0,452 \\
& $3-5$ & 0,384 & 0,472 & 0,501 \\
1998 & $6-10$ & 0,561 & 0,598 & 0,575 \\
& $>10$ & 0,538 & 0,646 & 0,541 \\
& $3-5$ & 0,612 & 0,703 & 0,623 \\
& $6-10$ & 0,407 & 0,489 & 0,473 \\
& $>10$ & 0,527 & 0,567 & 0,531 \\
\hline MÉDIA & & 0,585 & 0,664 & 0,609 \\
\hline
\end{tabular}

$\mathrm{GD}=$ graus-dia; $\mathrm{C}=$ chuva.
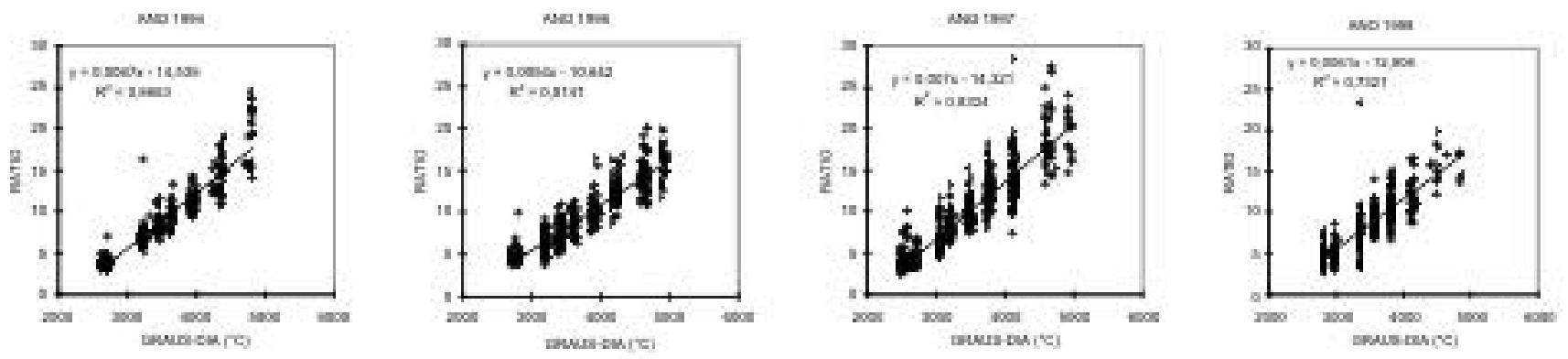

FIGURA 2 - Curvas da equação de regressão linear para o "ratio", em função de graus-dia acumulados por ano, em frutos da primeira florada de laranjeiras-'Valência' e 'Natal', com 6 a 10 anos de idade. Bebedouro-SP.
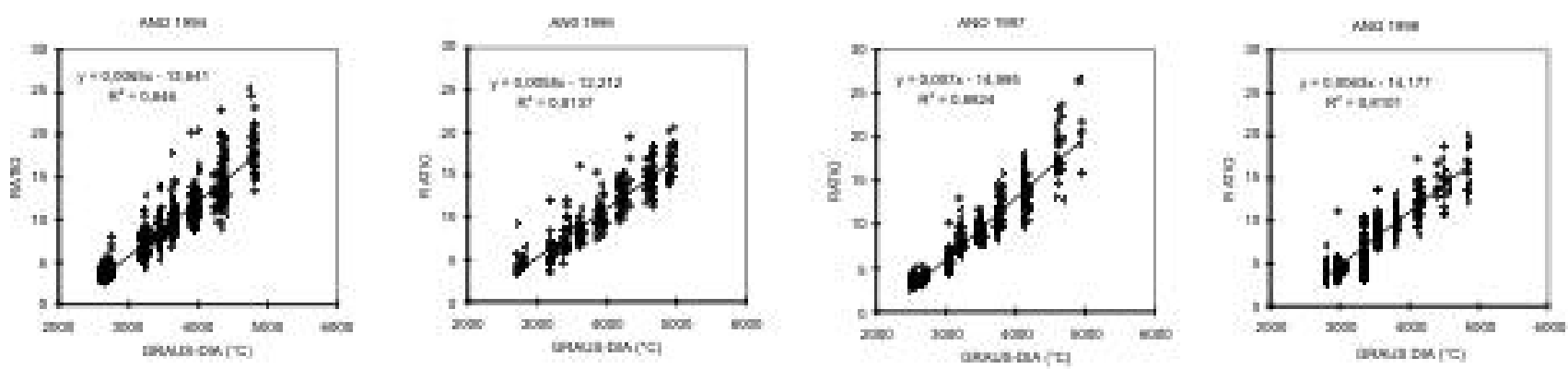

FIGURA 3 - Curvas da equação de regressão linear para o "ratio", em função de graus-dia acumulados por ano, em frutos da primeira florada de laranjeiras-'Valência' e 'Natal', com idade superior a 10 anos. Bebedouro-SP. 

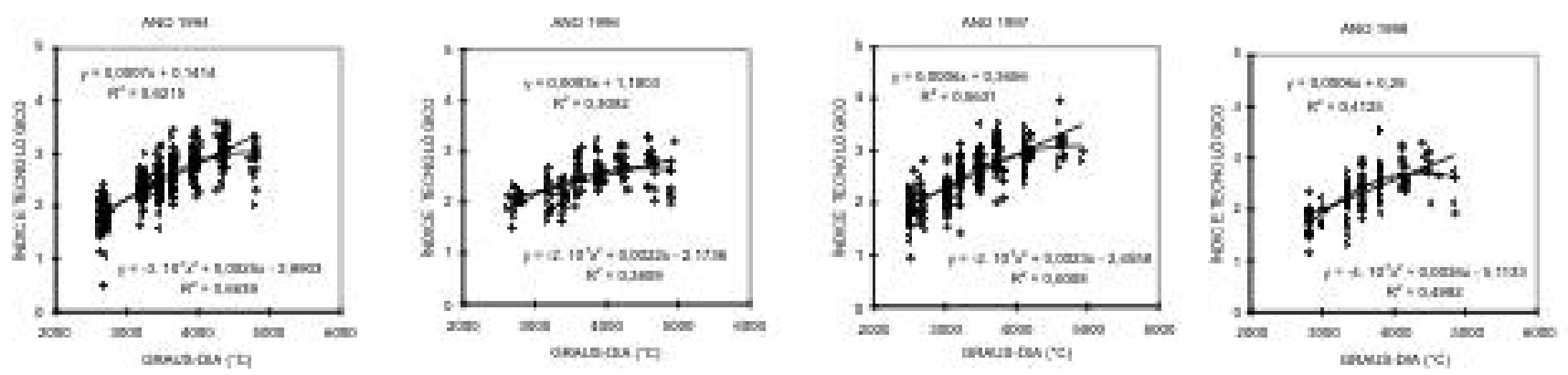

FIGURA 4 - Curvas das equações de regressão linear e quadrática para o índice tecnológico, em função de graus-dia acumulados por ano, em frutos da primeira florada de laranjeiras-'Valência' e 'Natal', com 3 a 5 anos de idade. Bebedouro-SP.
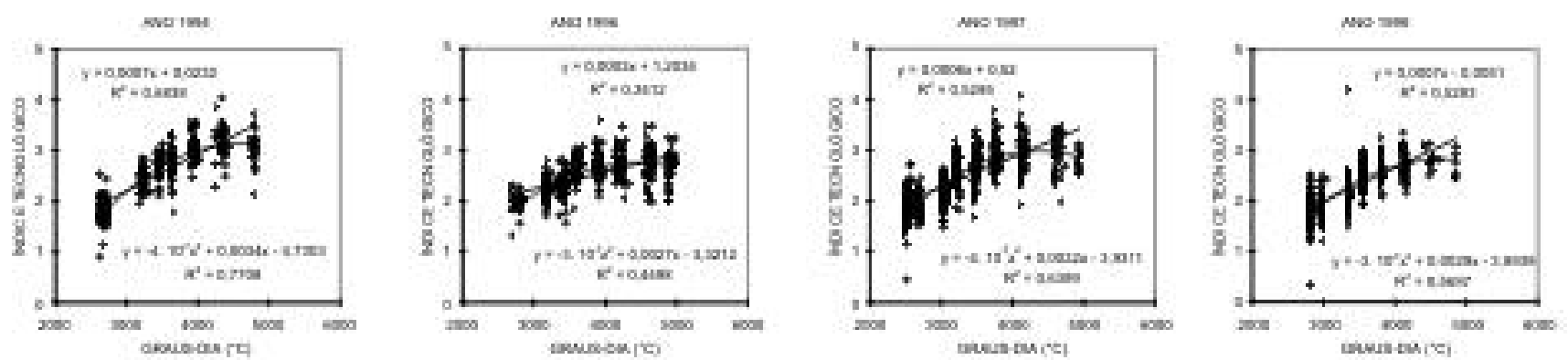

FIGURA 5 - Curvas das equações de regressão linear e quadrática para o índice tecnológico, em função de graus-dia acumulados por ano, em frutos da primeira florada de laranjeiras-'Valência' e 'Natal', com 6 a 10 anos de idade. Bebedouro-SP.
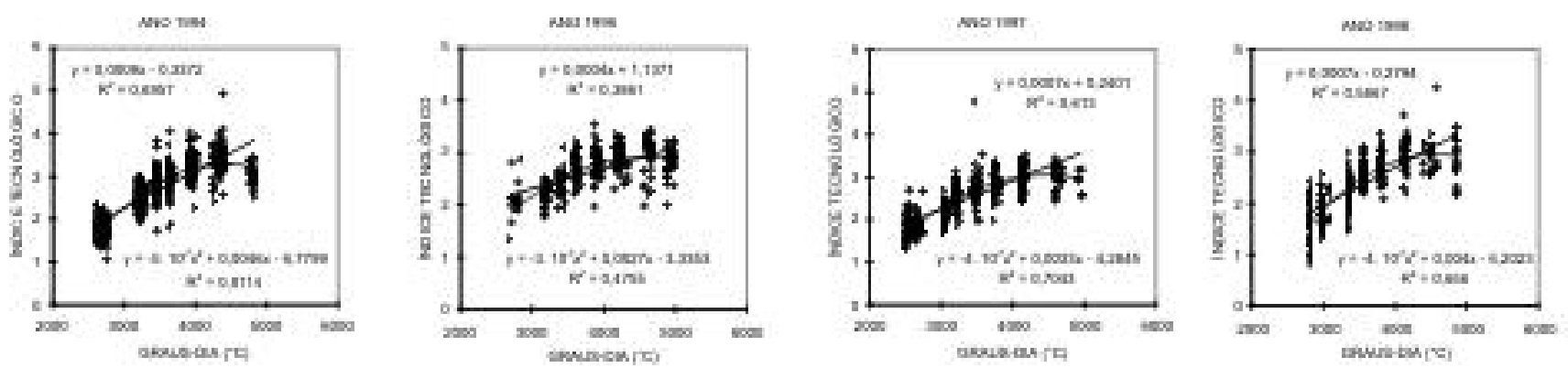

FIGURA 6 - Curvas das equações de regressão linear e quadrática para o índice tecnológico, em função de graus-dia acumulados por ano, em frutos da primeira florada de laranjeiras-'Valência' e 'Natal', com idade superior a 10 anos. Bebedouro-SP.

\section{CONCLUSÕES}

1. Para o índice tecnológico, a equação de regressão quadrática, com a variável independente graus-dia, acumulados a partir do florescimento, foi a que apresentou melhor ajuste, avaliado através do coeficiente de determinação ajustado. Para o "ratio", a equação de regressão linear simples foi a que mostrou melhor desempenho.

2. A inclusão da chuva não melhorou o ajuste das equações, indicando que a temperatura do ar, representada por grausdia, foi a variável que exerceu maior efeito na taxa de maturação dos frutos da $1^{\circ}$ florada de variedades de laranjas de maturação tardia, 'Natal' e 'Valência', na região de Bebedouro, durante os 4 anos estudados.

3. Os valores dos coeficientes de determinação obtidos e a falta de validação dos modelos não permitem que as equações de regressão obtidas sejam utilizadas como modelos de previsão.

\section{AGRADECIMENTOS}

Os autores agradecem à Coinbra-Frutesp, em nome do Eng $^{\circ} \mathrm{Agr}^{\circ}$ Ms Renato J. Marchi, por ter possibilitado a execução desse trabalho.

\section{REFERÊNCIAS BIBLIOGRÁFICAS}

ALBRIGO, G. Climatic influences on seasonal variation of Florida orange pounds solids. Proceedings International Society Horticulturae Science, Geneva, v. 2, p. 15-18, 1990.

CHEN, C.S. Models for seasonal changes in ${ }^{\circ}$ Brix and ratio of citrus fruit juice. Proceedings of the Florida State Horticulturae Society, Winten Haven, v. 103, p. 251-255, 1990. 
CHITARRA, M.I.F.; CAMPOS, M.A.P. Caracterização de alguns frutos cítricos cultivados em Minas Gerais. I- Laranjas doces comuns (Citrus sinensis L. Osbeck) em fase de maturação. In: CONGRESSO BRASILEIRO DE FRUTICULTURA, 6., 1981, Recife. Anais... Recife: Sociedade Brasileira de Fruticultura, 1981. p. 396-420.

CHITARRA, A.B.; CHITARRA, M.I.F. Componentes físicos e químicos da laranja Valência (Citrus sinensis L. Osbeck) em diversos estádios de maturação. In: CONGRESSO BRASILEIRO DE FRUTICULTURA, 5., 1979, Pelotas. Anais... Pelotas: Sociedade Brasileira de Fruticultura, 1979. v. 2, p. 563-586.

DI GIORGI, F; IDE, B.Y.; DIB, K.; MARCHI, R.J.; TRIBONI, H.R.; WAGNER, R.L.; ANDRADE, G. Influência do clima na produção de laranja. Laranja, Cordeirópolis, v. 1., p.163-192, 1991.

KIMBALL, D.A. Factors affecting the rate of maturation of citrus fruits. Proceedings of the Florida State Horticulturae Society, Winten Haven, v. 97, p. 40-44, 1984.

KIMBALL, D.A. Citrus processing: quality control and technology. New York: Van Nostrand, 1991. 473p.

MARCHI, R.J. Modelagem de curvas de maturação da laranjaPêra (Citrus sinensis L. Osbeck) na região de Bebedouro - SP. 1993. 107 f. Dissertação (Mestrado em Agronomia) - Faculdade de Ciências Agrárias e Veterinárias, Universidade Estadual Paulista, Jaboticabal, 1993

MONSELISE, S.P. Citrus. In: MONSELISE, S.P. (ed.) Handbook of fruit set and development. Florida, 1986. p.87-108.
NÚÑEZ, M.; GARCIA; M.E., ROSADO, F. Variaciones de los principais indicadores de la calidade de los frutos de tres especies de citricos. 11. Caracteristicas quimicas. Cultivos Tropicales, Havana, v. 9, n. 4, p. 71-75, 1987.

NÚÑEZ, M.; IGLESIAS, L. Relaciones entre los principales indicadores del crecimiento y la calidad interna de los frutos de naranja Valencia y algunas variables meteorologicas. Cultivos Tropicales, Havana, v. 12, n. 3, p. 57-61, 1991.

READ, J.B.; HENDRIX JR. C.M.; HENDRIX, D.L. Quality control manual for citrus processing plants. Florida: Intercit., 1986. $250 \mathrm{p}$.

SINCLAIR, W.B. The biochemistry and physiology of the lemon and other citrus fruits. Riverside: Univ. of California, 1984. 469 p.

SOULE, I., GRIERSON, W. Anatomy and physiology. In: WARDOWSHI, W.F., NAGY, S. (Ed.) Fresh citrus fruits. New York: 1986.p. 1-22.

VOLPE, C.A. Fenologia de citros. In: SEMINÁRIO INTERNACIONAL DE CITROS-FISIOLOGIA, 2., 1992, Bebedouro. Anais... Campinas: Fundação Cargill, 1992. p. 107-120.

VOLPE, C.A.; SCHÖFFEL, E.R.; BARBOSA, J.C. Influência de algumas variáveis meteorológicas sobre a qualidade dos frutos de laranjeiras-'Valência' e 'Natal'. Revista Brasileira de Agrometeorologia, Santa Maria, v. 8, n. 1, p. 85-94, 2000. 